\title{
Comparison between the long-axis/in-plane and short-axis/out-of-plane approaches for ultrasound-guided vascular catheterization: an updated meta-analysis and trial sequential analysis
}

This article was published in the following Dove Press journal:

Therapeutics and Clinical Risk Management

\section{Chao Liu* \\ Zhi Mao* \\ Hongjun Kang \\ Xin $\mathrm{Hu}$ \\ Shengmao Jiang \\ Pan $\mathrm{Hu}$ \\ Jie $\mathrm{Hu}$}

Feihu Zhou

Department of Critical Care Medicine, Chinese People's Liberation Army General Hospital, Beijing,

People's Republic of China

*These authors contributed equally to this work
Correspondence: Feihu Zhou

Department of Critical Care Medicine, Chinese People's Liberation Army General Hospital, National Clinical Research Center for Kidney Diseases, 28 Fu-Xing Road, Beijing 100853 ,

People's Republic of China

Tel +861066938148

Fax +86 I0 882I 9862

Email feihuzhou30I@I26.com
Background: A long-axis in-plane (LA-IP) approach and a short-axis out-of-plane (SA-OOP) approach are the two main approaches used in ultrasound (US)-guided vascular catheterization. However, the efficacy and safety of these approaches remain controversial. Therefore, we performed this meta-analysis to compare the two techniques in vascular catheterization.

Materials and methods: Relevant studies were searched in PubMed, Embase, and the Cochrane Library databases from database inception until August 2017. Randomized controlled trials comparing a long-axis approach with a short-axis approach for US-guided vascular cannulation were selected. The RevMan software was used to analyze the results, and trial sequential analysis (TSA) was further applied to determine whether the currently available evidence was sufficient and conclusive.

Results: Eleven studies met the inclusion criteria. Overall, 1,210 patients were included. The total success rate was similar between the SA-OOP and LA-IP approaches for US-guided vascular catheterization (risk ratio [RR], $1.01 ; 95 \% \mathrm{CI}, 0.99-1.04 ; P=0.35 ; I^{2}=48 \%$ ). In the radial artery (RA; RR, 1.00; 95\% CI, 0.96-1.05; $P=0.88 ; I^{2}=49 \%$ ) and internal jugular vein (IJV; RR, 1.00; 95\% CI, 0.98-1.02; $P=0.99 ; I^{2}=0 \%$ ) subgroups, the total success rate was also similar and was confirmed by the TSA. For populations with subclavian vein (SCV) and axillary vein catheterization, the SA-OOP approach showed a benefit for first-attempt success rate. No significant differences in first-attempt success rate, cannulation times, or complications were found between the two approaches.

Conclusion: Despite a similar total success rate between the SA-OOP approach and the LA-IP approach when used for RA and IJV catheterization (as confirmed by TSA), further robust well-designed trials are warranted to evaluate other outcomes. There is insufficient evidence to definitively state that the SA-OOP approach was superior to the LA-IP approach when used for SCV and axillary vein catheterization. High-quality trials are needed to confirm or refute this finding.

Keywords: ultrasound guidance, long-axis in-plane, short-axis out-of-plane, vascular catheterization, radial artery, internal jugular vein

\section{Background}

Ultrasound (US)-guided vascular cannulation has become more popular in recent years, and US is a proven tool to increase the success rate and decrease complications in a wide variety of vascular catheterization procedures. ${ }^{1}$ Numerous meta-analyses ${ }^{2-7}$ have demonstrated the advantage of US-guided vascular catheterization over a blind 
landmark approach. However, there is a controversy regarding which approach is preferable for the US probe, ie, the shortaxis out-of-plane (SA-OOP) approach or the long-axis in-plane (LA-IP) approach. Each approach has distinct advantages and disadvantages that may either increase or decrease complications. ${ }^{8}$ In the SA-OOP approach, the relationships of the target vessel to the adjacent vessels are visualized, but the needle tip may not be continuously visualized during catheter placement. In the LA-IP approach, the needle path including the tip can be continuously visualized during catheterization; however, the relationship of the target vessel to adjacent vessels may be lost. ${ }^{9}$ A previous meta-analysis of five studies showed that there is insufficient evidence to definitively determine which approach is superior in patients undergoing US-guided vascular catheterization. ${ }^{10}$ Several randomized controlled trials (RCTs) on this topic have been published recently. Therefore, we performed this updated meta-analysis of RCTs to compare the efficacy and safety of these two techniques and further applied trial sequential analysis (TSA) to determine whether the currently available evidence was sufficient and conclusive. This in turn can provide more reliable evidence for clinical practice.

\section{Materials and methods}

The Preferred Reporting Items for Systematic Reviews and Meta-Analyses (PRISMA statement) guidelines were used to perform this meta-analysis. ${ }^{11}$ Ethical approval was not required.

\section{Search strategy and study selection}

A search of the PubMed (US National Library of Medicine, Bethesda, MD, USA), Embase, and Cochrane Central Register of Controlled Trials databases was conducted from database inception to August 2017. Specific search strategies were developed for each database using exploded Medical Subject Headings and the appropriate corresponding keywords including "ultrasound," "ultrasonography," "ultrasonic" AND "catheterization," "cannulation," "catheter," "catheters," "insertion" AND "long axis," "short axis," "in plane," "out of plane," "longitudinal," "transverse" AND "RCTs." In addition, the reference lists of the screened full-text studies were searched to identify other potentially eligible trials. Searches were performed without language restrictions. A further search by manually reviewing conference proceedings and the references of review articles was performed.

The inclusion criteria were as follows: 1) population: adult patients requiring vascular catheterization; 2) comparison: the study compared LA-IP vs SA-OOP techniques for
US-guided vascular catheterization; 3 ) outcome measure: the total success rate was reported; and 4) study design: RCTs.

\section{Data extraction and outcome measurement}

Two investigators (CL and ZM) independently performed the study selection. When data were missing or incomplete, the original authors were contacted by written correspondence for clarification. Disagreements between the two investigators were resolved by consensus or adjudicated by a third investigator (FZ). The dynamic needle tip positioning (DNTP) technique was classified as the SA-OOP approach. One standard form was used for data collection, which included the following information: first author, year of publication, patient population, the number of patients, age of patients, total success rate, first-attempt success rate, cannulation time, number of attempts, experience of the operators, puncture site, and type of US equipment. The primary outcome was the total success rate, and secondary outcomes included the first-attempt success rate, cannulation time, and the number of attempts.

\section{Risk of bias assessment}

We assessed the internal validity of included trials using the Cochrane Collaboration Risk of Bias tool. ${ }^{12}$ The criteria in the tool included a description of the random sequence generation, allocation concealment, blinding of outcome assessment, incomplete outcome data, selective reporting, and other bias. Criteria were individually scored as high, low, or unclear risk of bias.

\section{Statistical analysis}

The statistical analysis was performed using RevMan software (version 5.3; Cochrane Collaboration, Copenhagen, Denmark) for outcome measurements. The results of the risk ratio (RR) for dichotomous outcomes or the mean difference (MD) for continuous data were expressed as means and 95\% CIs. A random-effects model was used regardless of heterogeneity, which was estimated using the $I^{2}$ statistic. A value of $I^{2}>50 \%$ indicated significant heterogeneity. ${ }^{13}$ Potential sources of heterogeneity were identified by sensitivity analyses. We further conducted subgroup analyses according to the puncture site. Publication bias was assessed using the Begg and Egger tests. $P<0.05$ was considered to indicate a statistically significant difference. The sample mean and SD were estimated according to the sample size, median, range, and/or interquartile range. ${ }^{14}$ 


\section{TSA}

An updated meta-analysis with new studies may result in false positive results; thus, to decrease the risk of type I errors, the TSA was used. This method can determine whether the present evidence is reliable and conclusive. ${ }^{15}$ When the cumulative Z-curve crosses the trial sequential monitoring boundary or enters the futility area, a sufficient level of evidence for the anticipated intervention effect may have been reached, and no further trials are needed. If the Z-curve does not cross any of the boundaries and the required information size (RIS) has not been reached, evidence to reach a conclusion is insufficient, and more trials are needed to confirm the results. For this TSA, we estimated the RIS using $\alpha=0.05$ (two sided), $\beta=0.10$ (power $90 \%$ ), the control event proportions calculated from the long-axis group, and a relative risk reduction of $10 \%$ in outcomes. TSA was conducted using TSA Version 0.9 Beta (www.ctu.dk/tsa). ${ }^{16}$

\section{Results}

\section{Study enrolment and characteristics}

A total of 348 potentially relevant studies were collected, of which 334 studies were excluded based on titles, abstracts, and duplicate studies. Three articles were excluded because the article type was a letter or meta-analysis. Ultimately, 11 studies $^{17-27}$ fulfilled the inclusion criteria for the metaanalysis (Figure 1). The study characteristics are summarized in Table 1. These studies were published between

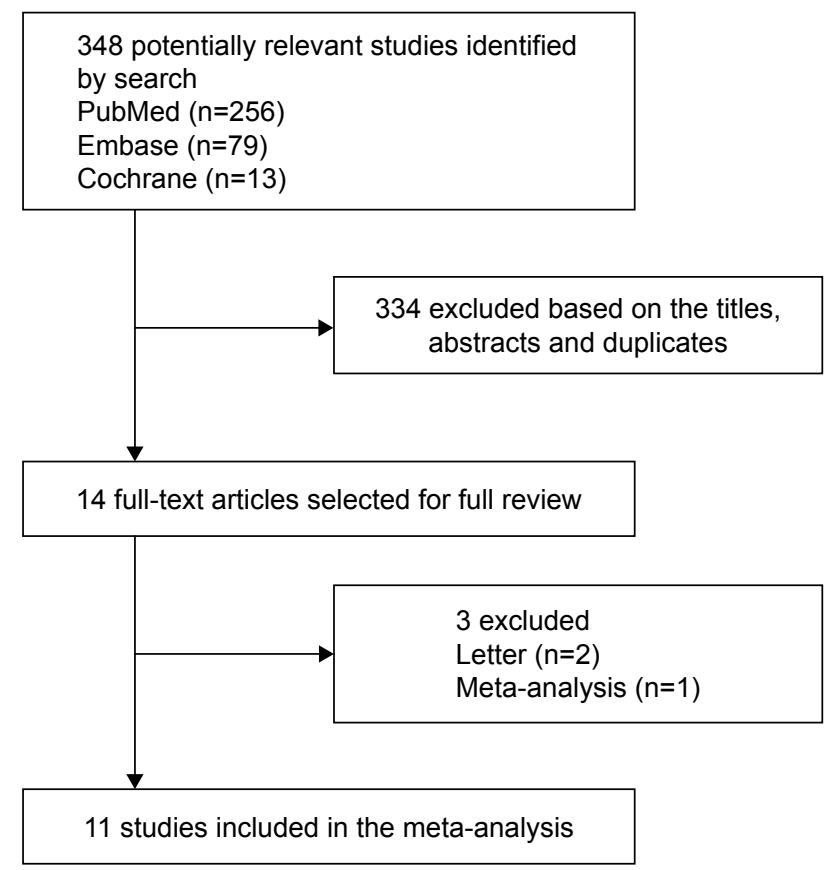

Figure I Flow chart of the study selection process.
2011 and 2017, and a total of 1,210 patients were included. The sample size of the studies ranged from 40 to 190 subjects. Baseline characteristics were similar between the two groups. All of the operators had experience in US-guided vascular catheterization. All studies reported the total success rate, and various complications were reported among these studies. The details for the risk of bias tool are shown in Figure 2. No study fulfilled all criteria for a low risk of bias. A randomized sequence was adequately generated in all studies. Due to the nature of the studies, it was impossible to perform the study blinded. No potential publication bias was observed for primary outcomes (data not shown).

\section{Primary outcome}

\section{Total success rate}

All studies investigated the total success rate of both the SA-OOP and LA-IP approaches for US-guided vascular catheterization. No significant difference was observed between the two groups (RR, 1.01; 95\% CI, 0.99-1.04; $P=0.35$; $I^{2}=48 \%$; Figure 3). Subgroup analyses were performed to investigate the source of heterogeneity. In the radial artery (RA) subgroup, ${ }^{19,21,26,27}$ the total success rate was no different between the SA-OOP and LA-IP groups (RR, 1.00; 95\% CI, $0.96-1.05 ; P=0.88 ; I^{2}=49 \%$; Figure 3 ), and the TSA confirmed this result (the cumulative Z-curve crossed the futility boundary and entered the futility area; Figure 4A). In the internal jugular vein (IJV) subgroup, ${ }^{17,20,22,23}$ the total success rate was also no different between the SA-OOP and LA-IP groups (RR, 1.00; 95\% CI, 0.98-1.02; $P=0.99$; $I^{2}=0 \%$; Figure 3 ) and the TSA also confirmed the result (the cumulative Z-curve crossed the futility boundary and entered the futility area; Figure 4B). Only one study ${ }^{25}$ was included in the subclavian vein ( $\mathrm{SCV}$ ) subgroup, and the results show that the SA-OOP approach was more efficacious than the LA-IP approach in increasing the total success rate (RR, 1.23; 95\% CI, 1.10-1.38; $P<0.01$; Figure 3).

\section{Secondary outcomes}

\section{First-attempt success rate}

Nine studies ${ }^{17,19,21-27}$ reported the first-attempt success rate. There was no significant difference between the LA-IP approach group and the SA-OOP approach group (RR, 1.14; 95\% CI, 0.96-1.35, $P=0.14, I^{2}=80 \%$; Figure 5). Due to remarkable heterogeneity, preset subgroup analyses were performed for the populations with RA catheterization (RR, $1.03 ; 95 \% \mathrm{CI}, 0.77-1.38 ; P=0.85, P=84 \%$; Figure 5 ) and IJV catheterization (RR, 1.12; 95\% CI, 0.84-1.49; $P=0.33$; $I^{2}=74 \%$; Figure 5). No significant differences were observed. 
Table I Characteristics of the II included trials

\begin{tabular}{|c|c|c|c|c|c|c|c|}
\hline Study & Site & $\begin{array}{l}\text { No of } \\
\text { patients } \\
\text { (M/F) }\end{array}$ & Patient population & $\begin{array}{l}\text { Mean age } \\
\text { (years) }\end{array}$ & Weight & $\begin{array}{l}\text { Total } \\
\text { success } \\
\text { rate }(\%)\end{array}$ & $\begin{array}{l}\text { First } \\
\text { attempt } \\
\text { success } \\
\text { rate (\%) }\end{array}$ \\
\hline $\begin{array}{l}\text { Sethi et al } \\
(2017 ; \text { India })^{27}\end{array}$ & RA & $\begin{array}{l}\text { S: } 75(46 / 29) \\
\text { L: } 75(4 I / 34)\end{array}$ & $\begin{array}{l}\text { Surgical patients } \\
\text { indicated for arterial } \\
\text { catheterization }\end{array}$ & $\begin{array}{l}\text { S: } 59.5 \pm 8.2^{\mathrm{a}} \\
\text { L: } 57.7 \pm 7.6^{\mathrm{a}}\end{array}$ & $\begin{array}{l}\text { S: } 62.8 \pm \text { I I.6 (kg) } \\
\text { L: } 64.6 \pm \text { I } 2.2(\mathrm{~kg})\end{array}$ & $\begin{array}{l}\text { S: } 92.0 \\
\text { L: } 93.3\end{array}$ & $\begin{array}{l}\text { S: } 80.0 \\
\text { L: } 82.7\end{array}$ \\
\hline $\begin{array}{l}\text { Abdalla et al } \\
(2017 ; \text { Egypt })^{26}\end{array}$ & RA & $\begin{array}{l}\text { S: } 42 \text { (NR) } \\
\text { L: } 42 \text { (NR) }\end{array}$ & $\begin{array}{l}\text { Surgical and ICU } \\
\text { patients indicated for } \\
\text { arterial catheterization }\end{array}$ & $\begin{array}{l}\text { S: } 55 \pm 11^{a} \\
L: 59 \pm 9^{a}\end{array}$ & 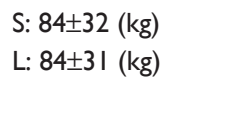 & $\begin{array}{l}\text { S: } 60 \\
\text { L: } 70\end{array}$ & $\begin{array}{l}\text { S: } 50 \\
\text { L: } 27\end{array}$ \\
\hline $\begin{array}{l}\text { Vezzani et al } \\
(2017 \text {; Italy })^{25}\end{array}$ & SCV & $\begin{array}{l}\text { S: } 95(74 / 2 \mathrm{I}) \\
\text { L: } 95(68 / 27)\end{array}$ & $\begin{array}{l}\text { Patients undergoing } \\
\text { cardiac surgery }\end{array}$ & $\begin{array}{l}S: 70 \pm 13^{\mathrm{a}} \\
L: 7 I \pm I 2^{\mathrm{a}}\end{array}$ & $\begin{array}{l}\text { S: } 26 \pm 4 \text { (BMI) } \\
\text { L: } 27 \pm 5 \text { (BMI) }\end{array}$ & $\begin{array}{l}\text { S: } 96 \\
\text { L: } 78\end{array}$ & $\begin{array}{l}\text { S: } 86 \\
\text { L: } 67\end{array}$ \\
\hline $\begin{array}{l}\text { Maddali et al } \\
(2017 ; \text { Oman })^{24}\end{array}$ & $\begin{array}{l}\text { Axillary } \\
\text { vein }\end{array}$ & $\begin{array}{l}\text { S: } 43 \text { (NR) } \\
\text { L: } 43 \text { (NR) }\end{array}$ & $\begin{array}{l}\text { Adult cardiac surgical } \\
\text { patients }\end{array}$ & $\begin{array}{l}\text { S: } 59.7 \pm 13.0^{\mathrm{a}} \\
\text { L: } 61.8 \pm 13.8^{\mathrm{a}}\end{array}$ & $\begin{array}{l}\text { S: } 27.4 \pm 5 . I \text { (BMI) } \\
\text { L: } 26.0 \pm 4.3 \text { (BMI) }\end{array}$ & $\begin{array}{l}\text { S: } 97.7 \\
\text { L: } 90.7\end{array}$ & $\begin{array}{l}\text { S: } 79.1 \\
\text { L: } 46.5\end{array}$ \\
\hline $\begin{array}{l}\text { Shrestha et al } \\
(2016 ; \text { Nepal })^{23}\end{array}$ & IJV & $\begin{array}{l}\text { S: 4I (23/I8) } \\
\text { L: } 41 \text { (30/II) }\end{array}$ & $\begin{array}{l}\text { Adult subjects } \\
\text { presenting for elective } \\
\text { surgery or in intensive } \\
\text { care unit }\end{array}$ & $\begin{array}{l}\text { S: } 59.7 \pm 22.0^{\mathrm{a}} \\
\mathrm{L}: 49.6 \pm 18.6^{\mathrm{a}}\end{array}$ & NR & $\begin{array}{l}\text { S: } 100 \\
\text { L: } 100\end{array}$ & $\begin{array}{l}\text { S: } 51.2 \\
\text { L: } 67.9\end{array}$ \\
\hline $\begin{array}{l}\text { Batllori et al } \\
(2016 \text {; Spain })^{22}\end{array}$ & IJV & $\begin{array}{l}\text { S: } 73(45 / 28) \\
\text { L: } 75(49 / 26)\end{array}$ & $\begin{array}{l}\text { Patients (aged } \\
18 \text { years or above) in } \\
\text { whom IJVC had been } \\
\text { indicated }\end{array}$ & $\begin{array}{l}\text { S: } 64.9 \\
\text { L: } 64.0\end{array}$ & $\begin{array}{l}\text { S: } 27.7 \pm 4.8 \text { (BMI) } \\
\text { L: } 27.3 \pm 4 . I \text { (BMI) }\end{array}$ & $\begin{array}{l}\text { S: } 97.3 \\
\text { L: } 97.3\end{array}$ & $\begin{array}{l}\text { S: } 69.9 \\
\text { L: } 52\end{array}$ \\
\hline $\begin{array}{l}\text { Quan et al } \\
(2014 ; \text { China })^{21}\end{array}$ & RA & $\begin{array}{l}\text { S: } 81 \text { (59/22) } \\
\text { L: } 82(64 / 18)\end{array}$ & $\begin{array}{l}\text { Undergo liver surgery } \\
\text { or splenic resection } \\
\text { under general } \\
\text { anesthesia }\end{array}$ & $\begin{array}{l}\text { S: } 49.2 \pm 8 . I^{\mathrm{a}} \\
\text { L: } 46.1 \pm 7.9^{\mathrm{a}}\end{array}$ & $\begin{array}{l}\text { S: } 76.4 \pm I 2.2(\mathrm{~kg}) \\
\text { L: } 72.1 \pm 10.5(\mathrm{~kg})\end{array}$ & $\begin{array}{l}\text { S: } 100 \\
\text { L: } 97.6\end{array}$ & $\begin{array}{l}\text { S: } 88.9 \\
\text { L: } 73.2\end{array}$ \\
\hline $\begin{array}{l}\text { Tammam et al } \\
(2013 ; \text { Egypt })^{20}\end{array}$ & IJV & $\begin{array}{l}\text { S: } 30(13 / 17) \\
\text { L: } 30(16 / 14)\end{array}$ & $\begin{array}{l}\text { Critical care and } \\
\text { hemodialysis patients }\end{array}$ & $\begin{array}{l}\text { S: } 56.0 \pm 14.5^{\mathrm{a}} \\
\text { L: } 51.3 \pm 15.3^{\mathrm{a}}\end{array}$ & $\begin{array}{l}\text { S: } 27.4 \mathrm{I} \pm 7.17 \text { (BMI) } \\
\text { L: } 28.15 \pm 8.20 \text { (BMI) }\end{array}$ & $\begin{array}{l}\text { S: } 100 \\
\text { L: } 100\end{array}$ & NR \\
\hline $\begin{array}{l}\text { Berk et al } \\
(2013 ; \text { Turkey })^{19}\end{array}$ & RA & $\begin{array}{l}\text { S: } 54(23 / 3 \mathrm{I}) \\
\text { L: } 54(30 / 24)\end{array}$ & $\begin{array}{l}\text { ASA I-III, require an } \\
\text { arterial catheter }\end{array}$ & $\begin{array}{l}\text { S: } 56 \pm I^{\mathrm{a}} \\
\text { L: } 54 \pm 2^{\mathrm{a}}\end{array}$ & $\begin{array}{l}\text { S: } 78 \pm 18(\mathrm{~kg}) \\
\text { L: } 76 \pm 16(\mathrm{~kg})\end{array}$ & $\begin{array}{l}\text { S: } 100 \\
\text { L: } 100\end{array}$ & $\begin{array}{l}\text { S: } 5 I \\
\text { L: } 76\end{array}$ \\
\hline $\begin{array}{l}\text { Mahler et al } \\
\text { (20I I; United } \\
\text { States) }{ }^{18}\end{array}$ & $\begin{array}{l}\text { Peripheral } \\
\text { intravenous }\end{array}$ & $\begin{array}{l}\text { S: } 20(6 / I 4) \\
\text { L: } 20(9 / I I)\end{array}$ & $\begin{array}{l}\text { Difficult IV access } \\
\text { patients in the ED }\end{array}$ & $\begin{array}{l}S: 48 \pm 15^{a} \\
L: 47 \pm 14^{a}\end{array}$ & $\begin{array}{l}\mathrm{C}: 20 \mathrm{I} \pm 54(\mathrm{lb}) \\
\mathrm{H}: 202 \pm 66(\mathrm{lb})\end{array}$ & $\begin{array}{l}\text { S: } 95 \\
\text { L: } 85\end{array}$ & NR \\
\hline $\begin{array}{l}\text { Chittoodan et al } \\
(201 \mathrm{I} \text {; Ireland })^{17}\end{array}$ & IJV & $\begin{array}{l}\text { S: } 49(37 / / 2) \\
\text { L: } 50(37 / / 3)\end{array}$ & $\begin{array}{l}\text { Adult subjects } \\
\text { presenting for elective } \\
\text { cardiac surgery }\end{array}$ & $\begin{array}{l}\text { S: } 62.9 \pm 13.2^{\mathrm{a}} \\
\mathrm{L}: 62.9 \pm 13 . \mathrm{I}^{\mathrm{a}}\end{array}$ & $\begin{array}{l}\text { S: } 85.2 \pm \text { I } 3.5(\mathrm{~kg}) \\
\text { L: } 84.1 \pm \text { I5.7 (kg) }\end{array}$ & $\begin{array}{l}\text { S: } 100 \\
\text { L: } 100\end{array}$ & $\begin{array}{l}\text { S: } 98 \\
\text { L: } 78\end{array}$ \\
\hline
\end{tabular}

Note: aean \pm SD.

Abbreviations: ASA, American Society of Anesthesiologists; BMI, body mass index; ED, emergency department; IJV, internal jugular vein; IJVC, internal jugular vein cannulation; L, long axis; NR, not reported; RA, radial artery; RCT, randomized controlled trials; S, short axis; SCV, subclavian vein. 


\begin{tabular}{|c|c|c|c|c|c|c|}
\hline $\begin{array}{l}\text { Cannulation } \\
\text { time }\end{array}$ & $\begin{array}{l}\text { No of } \\
\text { attempts }\end{array}$ & $\begin{array}{l}\text { No of } \\
\text { needle } \\
\text { redirections }\end{array}$ & Hematoma & $\begin{array}{l}\text { Arterial } \\
\text { puncture }\end{array}$ & Operator & Ultrasound equipment \\
\hline $\begin{array}{l}\text { S: } 28.4 \pm 8.2^{\mathrm{a}} \\
\text { L: } 27.6 \pm 7.6^{\mathrm{a}}\end{array}$ & NR & NR & $\begin{array}{l}\text { S: } 8 \\
\text { L: } 0\end{array}$ & NR & $\begin{array}{l}\text { Anesthetists had placed } \\
\text { more than } 100 \text { arterial lines } \\
\text { by using either in-plane or } \\
\text { out-of-plane approaches }\end{array}$ & $\begin{array}{l}\text { Probe (MicroMaxx }{ }^{\circledR} \\
\text { SLA/I3-6 MHz, SonoSite } \\
\text { Inc., Bothell, WA, USA), } \\
\text { ultrasound system (SonoSite } \\
\text { MicroMaxx }{ }^{\circledR} \text { Ultrasound } \\
\text { System, SonoSite Inc.) }\end{array}$ \\
\hline $\begin{array}{l}\text { S: } 28 \pm 19^{a} \\
L: 66 \pm 5^{a}\end{array}$ & $\begin{array}{l}\text { S: } 1.6 \pm 0.8^{a} \\
L: 1.8 \pm 0.7^{a}\end{array}$ & NR & $\begin{array}{l}\text { S: II } \\
\text { L: II }\end{array}$ & NR & Expert operators & $\begin{array}{l}\text { Toshiba Xario, Japan, } \\
\text { PLT-805AT transducer }\end{array}$ \\
\hline $\begin{array}{l}\text { S: } 69 \pm 74^{a} \\
\text { L: } 98 \pm 103^{a}\end{array}$ & NR & $\begin{array}{l}\text { S: } 0.39 \pm 0.88^{\mathrm{a}} \\
\mathrm{L}: 0.88 \pm 1.15^{\mathrm{a}}\end{array}$ & $\begin{array}{l}\text { S: } 0 \\
\text { L: I }\end{array}$ & $\begin{array}{l}\text { S: } 0 \\
\text { L: } 7\end{array}$ & $\begin{array}{l}\text { Anesthesiologists with } \\
\text { 3- and 6-year experience } \\
\text { in US-guided central vein } \\
\text { cannulation }\end{array}$ & $\begin{array}{l}\text { Philips CX50 system (Philips } \\
\text { Healthcare, Eindhoven, the } \\
\text { Netherlands) equipped with } \\
\text { a high-frequency, linear array } \\
\text { probe at } 10 \mathrm{MHz}\end{array}$ \\
\hline $\begin{array}{l}\text { S: } 4 \mid 4 \pm 228^{a} \\
L: 618 \pm 3 \mid 2^{a}\end{array}$ & $\begin{array}{l}\text { S: } 1.4 \pm 0.7^{a} \\
\text { L: } 1.8 \pm 0.9^{a}\end{array}$ & $\begin{array}{l}\text { S: } 1.3 \pm 0.9^{a} \\
L: 1.0 \pm 1.15^{a}\end{array}$ & $\begin{array}{l}\text { S: I } \\
\text { L: } 2\end{array}$ & $\begin{array}{l}\text { S: I } \\
\text { L: } 4\end{array}$ & Experienced anesthetists & $\begin{array}{l}\text { I2L-RS linear array } \\
\text { transducer from GE } \\
\text { (frequency range, 5-I5 MHz; } \\
\text { field of view, I92 mm) } \\
\text { compatible with a Logiq E } \\
\text { ultrasound machine } \\
\text { (GE Healthcare, Wauwatosa, } \\
\text { WI, USA) }\end{array}$ \\
\hline NR & NR & NR & NR & $\begin{array}{l}\text { S: I } \\
\text { L: I }\end{array}$ & $\begin{array}{l}\text { Experienced anesthetists } \\
\text { with a minimum of } 3 \text { years of } \\
\text { experience in cannulation of } \\
\text { central veins }\end{array}$ & $\begin{array}{l}\text { A 6-I0 L38 MHz linear } \\
\text { transducer SonoSite Turbo } \\
\text { unit (SonoSite, MicroMaxx, } \\
\text { Bothwell, WA, USA) }\end{array}$ \\
\hline $\begin{array}{l}\text { S: } 35.0 \pm 23.4^{\mathrm{a}} \\
\text { L: } 46.1 \pm 36.3^{\mathrm{a}}\end{array}$ & $\begin{array}{l}\text { S: } 1.51 \pm 0.97^{\mathrm{a}} \\
\text { L: } 1.92 \pm 1.36^{\mathrm{a}}\end{array}$ & NR & $\begin{array}{l}\text { S: I } \\
\text { L: } 2\end{array}$ & NR & $\begin{array}{l}\text { Anesthetists with appropriate } \\
\text { proficiency in ultrasound- } \\
\text { guided central venous access }\end{array}$ & $\begin{array}{l}\text { S-Nerve ultrasound machine } \\
\text { (SonoSite Inc.) with a } \\
\text { I3-6 MHz 38-mm linear array } \\
\text { probe was used }\end{array}$ \\
\hline $\begin{array}{l}\text { S: } 29.7 \pm 17.2^{\mathrm{a}} \\
\text { L: } 26.2 \pm 9.8^{\mathrm{a}}\end{array}$ & NR & NR & $\begin{array}{l}\text { S: } 12 \\
\text { L: } 15\end{array}$ & NR & $\begin{array}{l}\text { Experienced anesthetists, who } \\
\text { had previously cannulated } \\
450 \text { radial arteries and } \\
\text { used the ultrasound-guided } \\
\text { technique for approximately } \\
200 \text { procedures }\end{array}$ & $\begin{array}{l}\text { Terason } 2000^{+} ; \text {Terason, } \\
\text { Burlington, MA, USA }\end{array}$ \\
\hline S: $52.3 \pm\left. 11.9\right|^{\mathrm{a}}$ & S: $1.13 \pm 0.35^{\mathrm{a}}$ & NR & S: I & S: I & Well-trained attending & Portable unit (GE LogiqBook \\
\hline L: $52.7 \pm 11.74^{\mathrm{a}}$ & L: $1.17 \pm 0.38^{\mathrm{a}}$ & & L: 0 & L: 0 & $\begin{array}{l}\text { anesthetists, nephrologists, } \\
\text { or intensivists with similar } \\
\text { experience (at least } 5 \text { years } \\
\text { of experience in performing } \\
\text { ultrasound-guided method) }\end{array}$ & $\begin{array}{l}\text { XP Portable Ultrasound } \\
\text { Machine; General Electric } \\
\text { Company, GE Healthcare } \\
\text { - Americas, USA) and a } \\
\text { I0 MHz linear-array US probe }\end{array}$ \\
\hline S: $46.8 \pm 34^{\mathrm{a}}$ & S: $1.5 \pm 0.5^{a}$ & S: $2 \pm I^{\mathrm{a}}$ & S: 23 & NR & Anesthetists (had placed & Ultrasound system (Esaote \\
\hline L: $23.7 \pm 17^{a}$ & L: $1.27 \pm 0.4^{\mathrm{a}}$ & $\mathrm{L}: 1.5 \pm 0.6^{\mathrm{a}}$ & L: 2 & & $\begin{array}{l}\text { more than } 50 \text { ultrasound- } \\
\text { guided arterial lines) }\end{array}$ & $\begin{array}{l}\text { My Lab 30, US Machine, } \\
\text { Florence, Italy) and a linear } \\
\text { probe with } 18 \mathrm{MHz} \text { frequency }\end{array}$ \\
\hline $\begin{array}{l}\text { S: } 34(35)^{c} \\
\text { L: } 96(59)^{c}\end{array}$ & $\begin{array}{l}\text { S: } 1.5 \pm 0.7^{\mathrm{a}} \\
\text { L: } 1.4 \pm 0.7^{\mathrm{a}}\end{array}$ & NR & NR & NR & $\begin{array}{l}\text { ED attending physicians } \\
\text { and nurses (more than } \\
20 \text { ultrasound-guided } \\
\text { peripheral or central lines) }\end{array}$ & $\begin{array}{l}\text { Single-operator technique } \\
\text { and 5-10 MHz, L38 probe } \\
\text { on a SonoSite MicroMaxx; } \\
\text { SonoSite Inc. }\end{array}$ \\
\hline $\begin{array}{l}\text { S: } 39.6 \pm 18.4^{\mathrm{a}} \\
\text { L: } 46.9 \pm 42.4^{\mathrm{a}}\end{array}$ & $\begin{array}{l}\text { S: } 1.02 \pm 0.2^{\mathrm{a}} \\
\text { L: } 1.24 \pm 0.56^{\mathrm{a}}\end{array}$ & NR & NR & $\begin{array}{l}\text { S: } 0 \\
\text { L: } 2\end{array}$ & $\begin{array}{l}\text { Anesthetists (more than } \\
50 \text { ultrasound-guided internal } \\
\text { jugular cannulations) }\end{array}$ & $\begin{array}{l}\text { A 6-I0 L38 MHz linear } \\
\text { transducer SonoSite } \\
\text { Titanunit (SonoSite, } \\
\text { MicroMaxx; SonoSite Inc.) }\end{array}$ \\
\hline
\end{tabular}




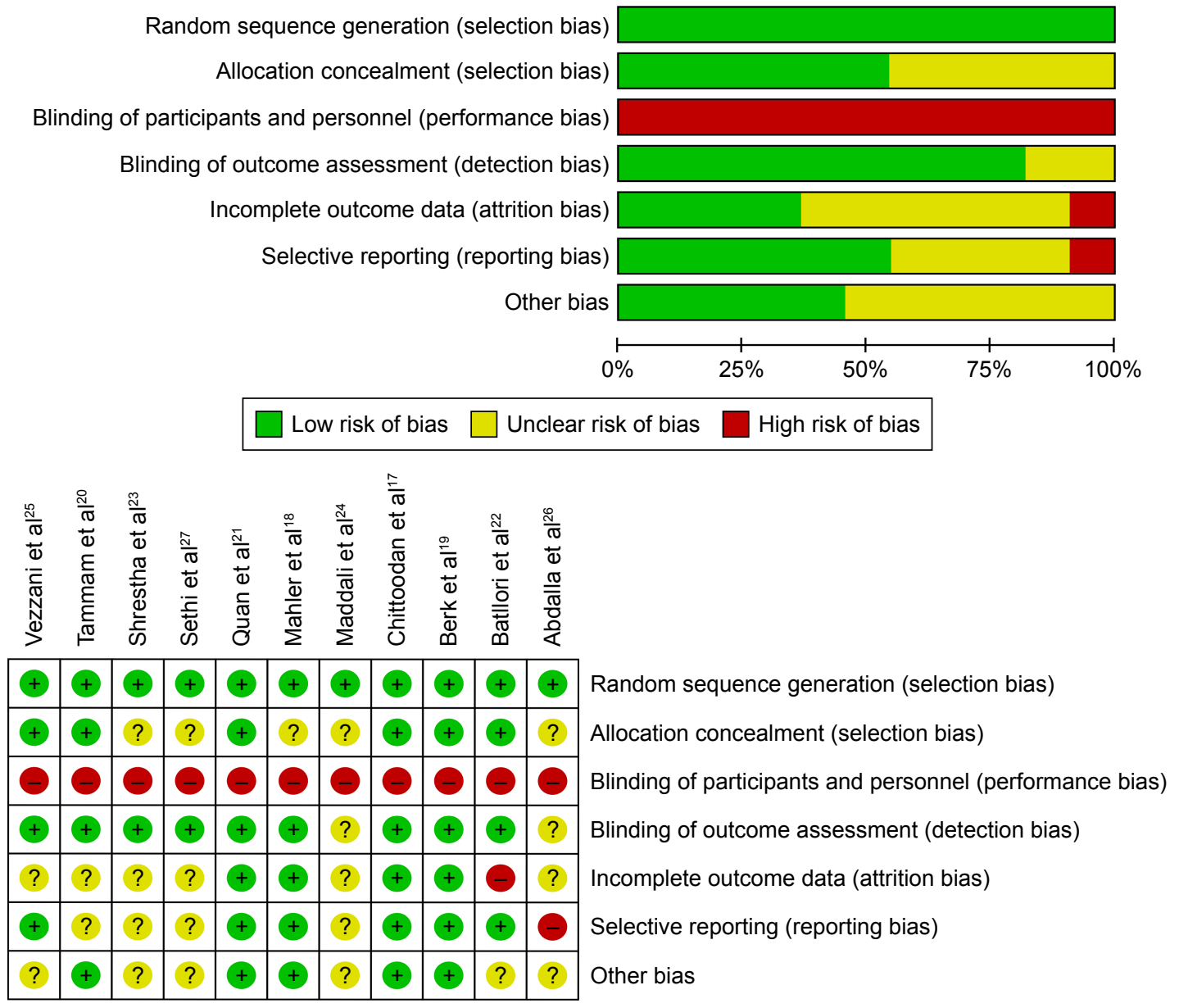

Figure 2 Assessment for risk of bias.

For populations with $\mathrm{SCV}$ catheterization ${ }^{25}$ and axillary vein catheterization, ${ }^{24}$ the SA-OOP approach showed a benefit.

Other secondary outcomes are summarized in Table 2, including cannulation times, the number of attempts, complications of hematoma, and arterial puncture. The cannulation times of the two approaches used in the US-guided RA and IJV catheterization were similar. The number of attempts may be reduced when the SA-OOP approach is used in US-guided IJV catheterization and the LA-IP approach is used in US-guided RA catheterization. No significant differences were observed when comparing the complications of hematoma and arterial puncture between the two approaches.

\section{Discussion}

The key findings of this updated meta-analysis are as follows. 1) The total success rate was similar between the SA-OOP approach and LA-IP approach when used for RA and IJV catheterization, as confirmed by TSA. The SA-OOP approach showed a benefit when used for SCV catheterization; however, further RCTs are needed to confirm this. 2) The first-attempt success rate was also similar between the two approaches when used for US-guided RA or IJV catheterization. Whether the SA-OOP approach has an advantage in increasing the first-attempt success rate when used for SCV and axillary vein catheterization also requires further studies to confirm or refute this finding. 3) The number of attempts may be reduced when the SA-OOP approach is used in US-guided IJV catheterization and when the LA-IP approach is used in US-guided RA catheterization. 4) No significant differences in cannulation times or complications were found between the two approaches.

There are several differences between our meta-analysis and a previous meta-analysis. ${ }^{10}$ First, this meta-analysis included an additional six studies. ${ }^{22-27}$ Thus, this updated metaanalysis represents the latest and most comprehensive study. Second, TSA was further applied to better establish sufficient and conclusive evidence. Third, we also analyzed the difference between the two approaches when used in different vessels.

In this meta-analysis, the total success rate was similar when the two approaches were used for US-guided RA and IJV catheterization and was confirmed by TSA. Moreover, more studies are needed to further evaluate whether the 


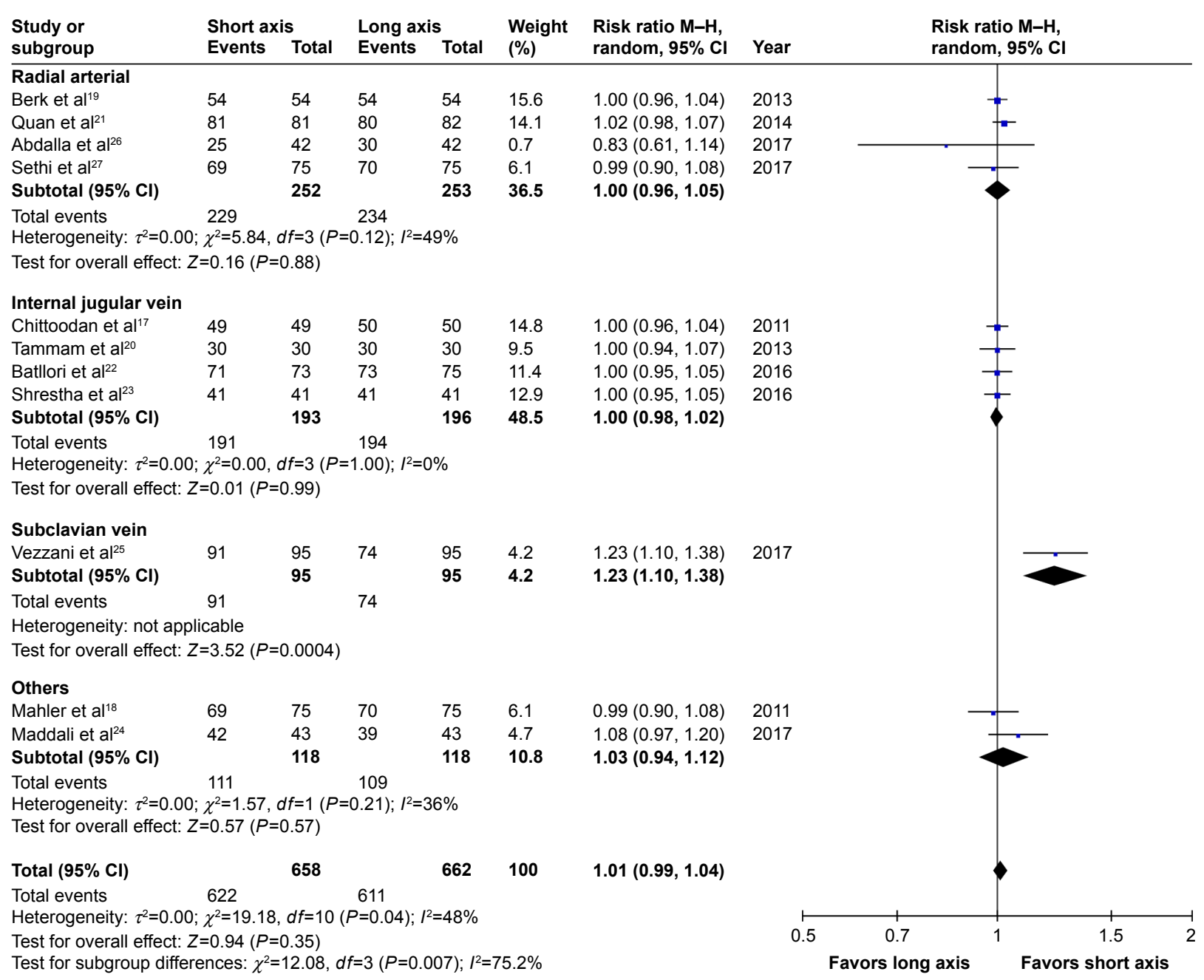

Figure 3 Forest plot of total success rate.

Abbreviation: $\mathrm{M}-\mathrm{H}$, Mantel-Haenszel.

complications, first-attempt success rate, and cannulation times are different between the two approaches. A study ${ }^{25}$ included in this meta-analysis showed that the SA-OOP approach may increase the total success rate when used for SCV catheterization; however, this led to controversy. ${ }^{28}$ Highquality studies are needed to confirm or refute this finding.

One study ${ }^{26}$ included in this meta-analysis reported that the oblique approach for US-guided RA catheterization may replace the two classic approaches due to its superior success rate, higher first-attempt success, and shorter time consumed for catheterization with higher operator satisfaction after the procedure. Confirmation of this requires further research. Another study ${ }^{22}$ in this meta-analysis compared three transducer orientation approaches for US-guided IJV catheterization and found that the oblique-axis (OAX) approach showed a higher first-needle pass success rate than LA-IP approach and a lower mechanical complications rate than the SA-OOP approach. Although more clinical studies are needed, this new approach may be superior in reducing complications related to IJV catheterization.
US-guidance for vascular catheterization is a userdependent technology, and it is necessary to use medical simulators to train novice US users before they practice on patients. However, there are some discrepancies between the medical simulator result and the patient result. For example, one study ${ }^{29}$ evaluated the two approaches used in SCV catheterization in a human torso mannequin and found that the LA-IP view for SCV catheterization was more efficient with a decreased time to cannulation and fewer redirections. The simulators could not reflect the actual circumstances of the patients, and they lacked the nerves and arteries from soft tissue that commonly complicate US-guided vascular catheterization. ${ }^{30}$ Hence, whether the benefit obtained from the medical simulators can be proven in live patients is unknown. Therefore, to provide more reliable evidence for clinical practice, more high-quality, anatomically accurate, vascular phantoms and standardized performance procedures are needed for US training. ${ }^{31}$ Furthermore, when using the medical simulator to train novice US users, we should carefully 

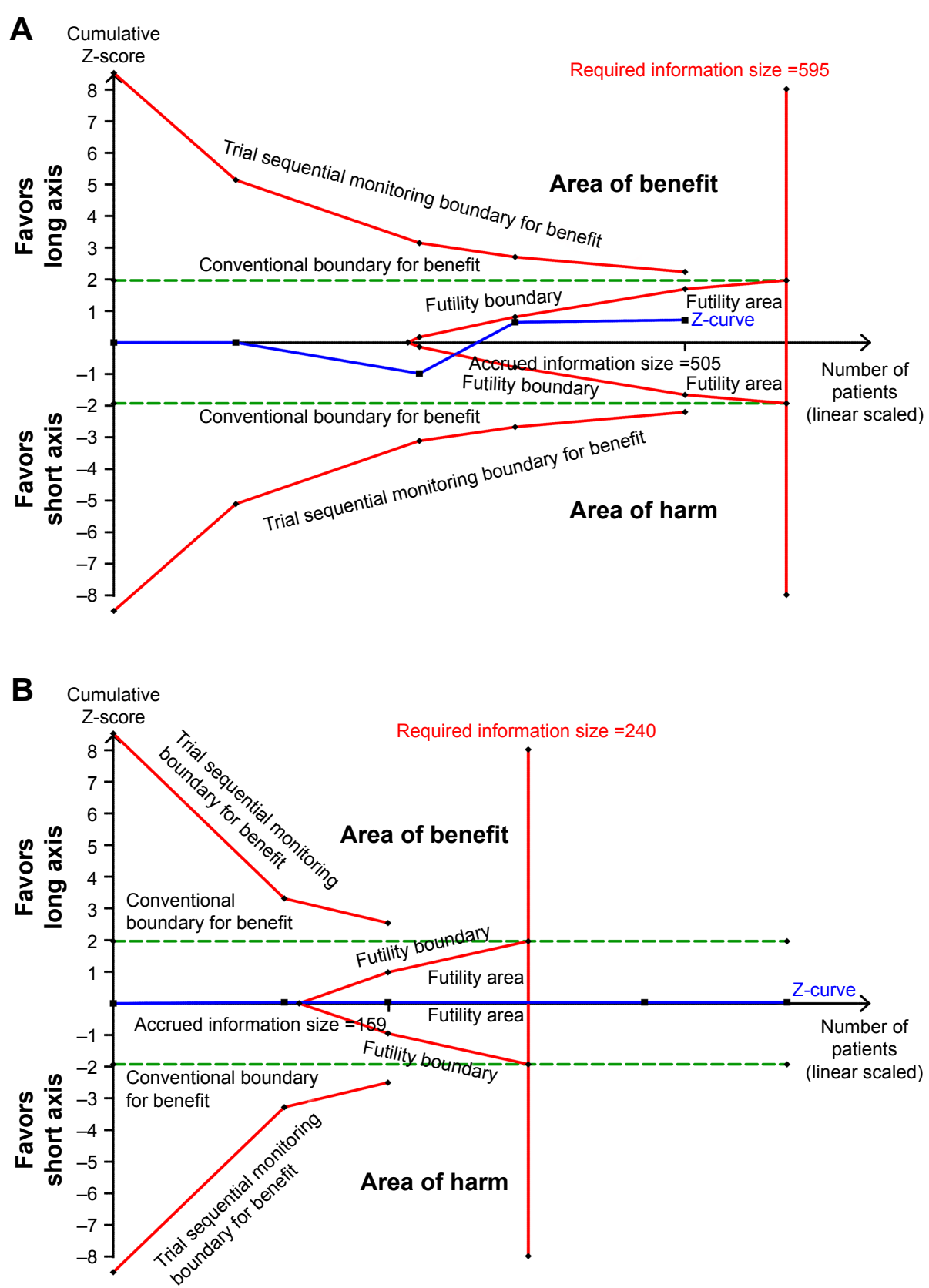

Figure 4 TSA result.

Notes: (A) RA subgroup. The DL approach of TSA used for the total success rate. A diversity-adjusted information size of 595 participants was calculated on the basis of a total success rate of $92.5 \%$ in the long-axis group with a relative risk reduction of $10 \%, \alpha=5 \%$ (two sided), $\beta=10 \%$ (power $90 \%$ ), and $l^{2}=0 \%$. The solid blue line represents a cumulative Z-curve, which crossed the futility boundary (complete red line) and reached the futility area. (B) IJV subgroup. The DL approach of TSA was used for the total success rate. A diversity-adjusted information size of 240 participants was calculated on the basis of a total success rate of $99 \%$ in the long-axis group with a relative risk reduction of $10 \%, \alpha=5 \%$ (two sided), $\beta=10 \%$ (power $90 \%$ ), and $R^{2}=0 \%$. The complete blue line represents the cumulative Z-curve, which entered the futility area and crossed the required information size boundary.

Abbreviations: DL, DerSimonian-Laird; IJV, internal jugular vein; M-H, Mantel-Haenszel; RA, radial artery; TSA, trial sequential analysis.

consider the discrepancy between the medical simulator and the patient to improve clinical practice.

This meta-analysis has several potential limitations. First, considerable heterogeneity was observed between the included studies with respect to blood vessel type, operator experience, US equipment, and outcome definition, which could have influenced the results; second, due to the limited data, it is difficult to perform more subgroup or sensitivity analyses. Third, double blinding was not performed due to the features of the trials, which may result in performance and detection bias.

\section{Conclusion}

A similar total success rate between the SA-OOP approach and the LA-IP approach for RA and IJV catheterization was confirmed by TSA. However, further robust and well-designed trials are warranted to evaluate other outcomes. There is 


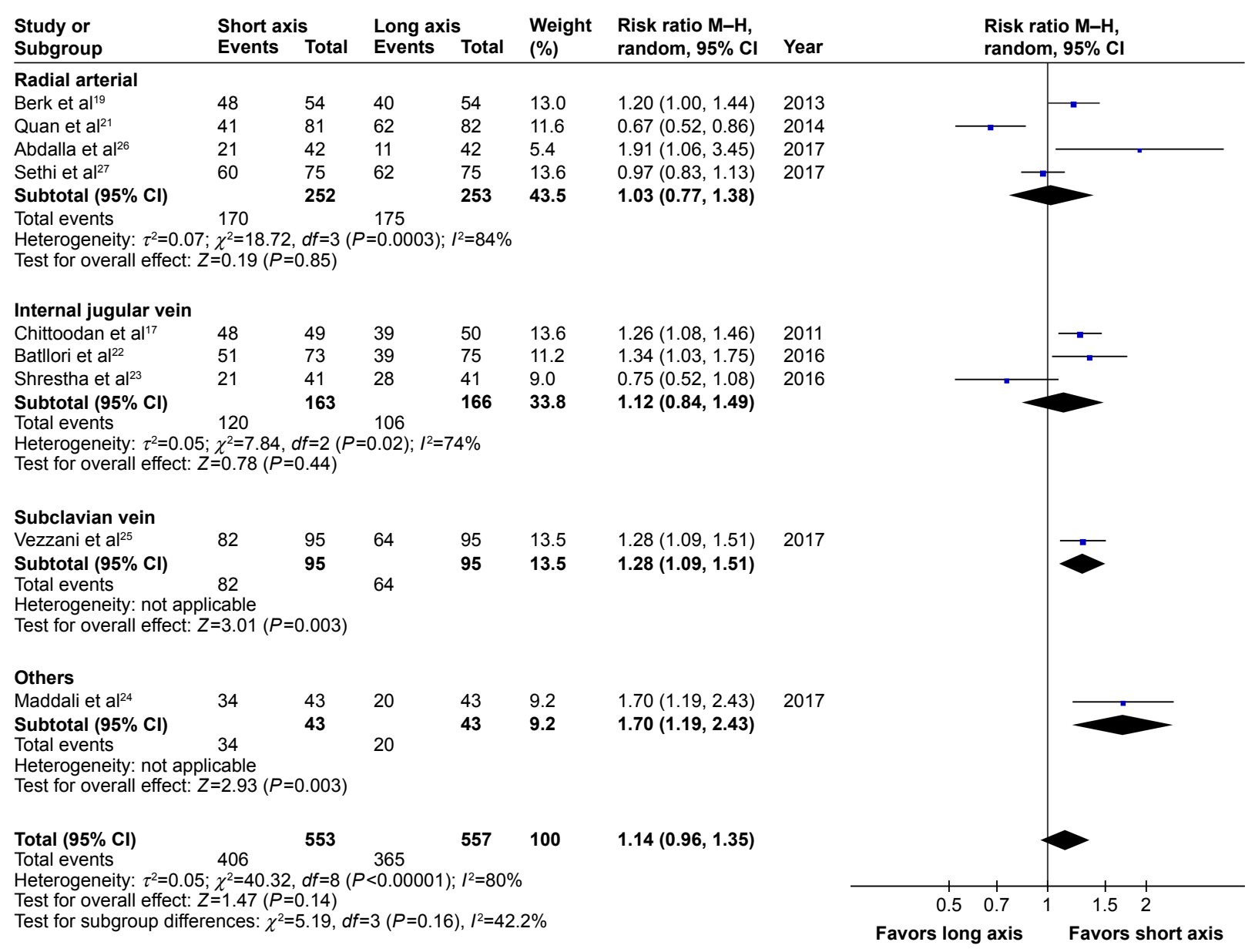

Figure 5 Forest plot of the first attempt success rate.

Abbreviation: $\mathrm{M}-\mathrm{H}$, Mantel-Haenszel.

insufficient evidence to definitively state that the SA-OOP approach was better than the LA-IP approach when used for SCV and axillary vein catheterization. High-quality trials are needed to confirm or refute this finding.

\section{Data sharing statement}

The authors confirm that all data underlying the findings are fully available without restriction. All relevant data are within the paper.

Table 2 Secondary outcomes

\begin{tabular}{|c|c|c|c|c|c|c|}
\hline & \multirow{2}{*}{$\begin{array}{l}\text { No of } \\
\text { studies }\end{array}$} & \multicolumn{2}{|c|}{ No of patients } & \multirow[t]{2}{*}{ MD (95\% Cl) } & \multirow{2}{*}{$\begin{array}{l}\text { Heterogeneity } \\
I^{2}(P \text {-value })\end{array}$} & \multirow{2}{*}{$\begin{array}{l}\text { Test for effect } \\
(P \text {-value) }\end{array}$} \\
\hline & & SA & $\overline{\text { LA }}$ & & & \\
\hline Cannulation & $3^{17,20,22, \mathrm{a}}$ & 152 & 155 & $-5.15(-12.28$ to 1.97$)$ & $45 \%(<0.01)$ & 0.16 \\
\hline time & $4^{19,21,26,27, \mathrm{~b}}$ & 252 & 253 & $-2.93(-21.10$ to 15.25$)$ & $98 \%(<0.01)$ & 0.75 \\
\hline \multirow{5}{*}{$\begin{array}{l}\text { Number of } \\
\text { attempts }\end{array}$} & $5^{17-20,22,24,26, c}$ & 311 & 314 & $-0.12(-0.30$ to 0.07$)$ & $74 \%(<0.01)$ & 0.22 \\
\hline & $3^{17,20,22, a}$ & 152 & 155 & $-0.18(-0.35$ to 0.00$)$ & $47 \%(0.15)$ & 0.04 \\
\hline & $I^{19,6}$ & 54 & 54 & 0.23 (0.06 to 0.40$)$ & Not applicable & $<0.01$ \\
\hline & \multirow{2}{*}{$\begin{array}{l}\text { No of } \\
\text { studies }\end{array}$} & \multicolumn{2}{|c|}{ No of patients } & RR (95\% CI) & Heterogeneity & Test for effect \\
\hline & & $\overline{\text { SA }}$ & $\overline{\text { LA }}$ & & $I^{2}(P$-value $)$ & (P-value) \\
\hline \multirow[t]{3}{*}{ Hematoma } & $6^{19-22,26,27, c}$ & 355 & 358 & 2.02 (0.68 to 5.98$)$ & $73 \%(<0.01)$ & 0.20 \\
\hline & $2^{19,21, \mathrm{a}}$ & 103 & 105 & $0.97(0.15$ to 6.50$)$ & $0 \%(0.38)$ & 0.98 \\
\hline & $4^{20,22,26,27, \mathrm{~b}}$ & 252 & 253 & 2.49 (0.66 to 9.37$)$ & $83 \%(<0.01)$ & 0.18 \\
\hline Arterial & $5^{17,20,23-25, c}$ & 258 & 259 & $0.36(0.1 \mathrm{I}$ to $\mathrm{I} .2 \mathrm{I})$ & $0 \%(0.4 I)$ & 0.10 \\
\hline puncture & $3^{17,20,23, \mathrm{a}}$ & 120 & 121 & $0.83(0.15$ to 4.55$)$ & $0 \%(0.47)$ & 0.83 \\
\hline
\end{tabular}

Notes: anternal jugular vein subgroup. ${ }^{\text {} R a d i a l ~ a r t e r y ~ s u b g r o u p . ~ ' A l l ~ s t u d i e s . ~}$

Abbreviations: LA, long axis; MD, mean difference; RR, relative risk; SA, short axis. 


\section{Acknowledgments}

This work was not supported by any funding.

\section{Disclosure}

The authors report no conflicts of interest in this work.

\section{References}

1. Moore CL. Ultrasound first, second, and last for vascular access. J Ultrasound Med. 2014;33(7):1135-1142.

2. Randolph AG, Cook DJ, Gonzales CA, Pribble CG. Ultrasound guidance for placement of central venous catheters: a meta-analysis of the literature. Crit Care Med. 1996;24(12):2053-2058.

3. Hind D, Calvert N, McWilliams R, et al. Ultrasonic locating devices for central venous cannulation: meta-analysis. BMJ. 2003; 327(7411):361.

4. Gu WJ, Tie HT, Liu JC, Zeng XT. Efficacy of ultrasound-guided radial artery catheterization: a systematic review and meta-analysis of randomized controlled trials. Crit Care. 2014;18(3):R93.

5. Gao YB, Yan JH, Gao FQ, Pan L, Wang XZ, Lv CJ. Effects of ultrasound-guided radial artery catheterization: an updated metaanalysis. Am J Emerg Med. 2015;33(1):50-55.

6. Lalu MM, Fayad A, Ahmed O, et al. Ultrasound-guided subclavian vein catheterization: a systematic review and meta-analysis. Crit Care Med. 2015;43(7):1498-1507.

7. Stolz LA, Stolz U, Howe C, Farrell IJ, Adhikari S. Ultrasound-guided peripheral venous access: a meta-analysis and systematic review. J Vasc Access. 2015;16(4):321-326.

8. Erickson CS, Liao MM, Haukoos JS, et al. Ultrasound-guided small vessel cannulation: long-axis approach is equivalent to short-axis in novice sonographers experienced with landmark-based cannulation. West J Emerg Med. 2014;15(7):824-830.

9. Dilisio R, Mittnacht AJ. The "medial-oblique" approach to ultrasoundguided central venous cannulation - maximize the view, minimize the risk. J Cardiothorac Vasc Anesth. 2012;26(6):982-984.

10. Gao YB, Yan JH, Ma JM, et al. Effects of long axis in-plane vs short axis out-of-plane techniques during ultrasound-guided vascular access. Am J Emerg Med. 2016;34(5):778-783.

11. Moher D, Liberati A, Tetzlaff J, Altman DG, Group P. Preferred reporting items for systematic reviews and meta-analyses: the PRISMA statement. Int J Surg. 2010;8(5):336-341.

12. Higgins JP, Altman DG, Gotzsche PC, et al. The Cochrane collaboration's tool for assessing risk of bias in randomised trials. BMJ. 2011; 343:d5928.

13. Higgins JP, Thompson SG, Deeks JJ, Altman DG. Measuring inconsistency in meta-analyses. BMJ. 2003;327(7414):557-560.

14. Wan X, Wang W, Liu J, Tong T. Estimating the sample mean and standard deviation from the sample size, median, range and/or interquartile range. BMC Med Res Methodol. 2014;14:135.

15. Liu C, Mao Z, Kang H, Hu J, Zhou F. Regional citrate versus heparin anticoagulation for continuous renal replacement therapy in critically ill patients: a meta-analysis with trial sequential analysis of randomized controlled trials. Crit Care. 2016;20(1):144
16. Thorlund K, Engstrøm J, Wetterslev J, Brok J, Imberger G, Gluud C [webpage on the Internet]. User Manual for Trial Sequential Analysis (TSA). Copenhagen, Denmark: Copenhagen Trial Unit, Centre for Clinical Intervention Research; 2011:1-115. Available from: www. ctu.dk/tsa. Accessed May 3, 2017.

17. Chittoodan S, Breen D, O’Donnell BD, Iohom G. Long versus short axis ultrasound guided approach for internal jugular vein cannulation: a prospective randomised controlled trial. Med Ultrason. 2011; 13(1):21-25.

18. Mahler SA, Wang H, Lester C, Skinner J, Arnold TC, Conrad SA. Shortvs long-axis approach to ultrasound-guided peripheral intravenous access: a prospective randomized study. Am J Emerg Med. 2011;29(9): 1194-1197.

19. Berk D, Gurkan Y, Kus A, Ulugol H, Solak M, Toker K. Ultrasoundguided radial arterial cannulation: long axis/in-plane versus short axis/ out-of-plane approaches? J Clin Monit Comput. 2013;27(3):319-324.

20. Tammam TF, El-Shafey EM, Tammam HF. Ultrasound-guided internal jugular vein access: comparison between short axis and long axis techniques. Saudi J Kidney Dis Transpl. 2013;24(4):707-713.

21. Quan Z, Tian M, Chi P, Cao Y, Li X, Peng K. Modified short-axis out-of-plane ultrasound versus conventional long-axis in-plane ultrasound to guide radial artery cannulation: a randomized controlled trial. Anesth Analg. 2014;119(1):163-169.

22. Batllori M, Urra M, Uriarte E, et al. Randomized comparison of three transducer orientation approaches for ultrasound guided internal jugular venous cannulation. Br J Anaesth. 2016;116(3):370-376.

23. Shrestha GS, Gurung A, Koirala S. Comparison between long- and short-axis techniques for ultrasound-guided cannulation of internal jugular vein. Ann Card Anaesth. 2016;19(2):288-292.

24. Maddali MM, Arora NR, Chatterjee N. Ultrasound guided out-ofplane versus in-plane transpectoral left axillary vein cannulation. J Cardiothorac Vasc Anesth. 2017;31(5):1707-1712.

25. Vezzani A, Manca T, Brusasco C, et al. A randomized clinical trial of ultrasound-guided infra-clavicular cannulation of the subclavian vein in cardiac surgical patients: short-axis versus long-axis approach. Intensive Care Med. 2017;43(11):1594-1601.

26. Abdalla UE, Elmaadawey A, Kandeel A. Oblique approach for ultrasound-guided radial artery catheterization vs transverse and longitudinal approaches, a randomized trial. J Clin Anesth. 2017;36:98-101.

27. Sethi S, Maitra S, Saini V, Samra T, Malhotra SK. Comparison of shortaxis out-of-plane versus long-axis in-plane ultrasound-guided radial arterial cannulation in adult patients: a randomized controlled trial. J Anesth. 2017;31(1):89-94.

28. Preda G, Bige N, Bonsey M, et al. Discussion on "a randomized clinical trial of ultrasound-guided infra-clavicular cannulation of the subclavian vein in cardiac surgical patients: short-axis versus long-axis approach". Intensive Care Med. 2017;43(8):1169-1170.

29. Vogel JA, Haukoos JS, Erickson CL, et al. Is long-axis view superior to short-axis view in ultrasound-guided central venous catheterization? Crit Care Med. 2015;43(4):832-839.

30. Resnick JR, Cydulka R, Jones R. Comparison of two transducers for ultrasound-guided vascular access in long axis. J Emerg Med. 2007; 33(3):273-276

31. AIUM practice guideline for the use of ultrasound to guide vascular access procedures. J Ultrasound Med. 2013;32(1):191-215.
Therapeutics and Clinical Risk Management

\section{Publish your work in this journal}

Therapeutics and Clinical Risk Management is an international, peerreviewed journal of clinical therapeutics and risk management, focusing on concise rapid reporting of clinical studies in all therapeutic areas, outcomes, safety, and programs for the effective, safe, and sustained use of medicines. This journal is indexed on PubMed Central, CAS,

\section{Dovepress}

EMBase, Scopus and the Elsevier Bibliographic databases. The manuscript management system is completely online and includes a very quick and fair peer-review system, which is all easy to use. Visit http://www.dovepress.com/testimonials.php to read real quotes from published authors. 